In-situ child and adolescent mental health simulation with human factors feedback delivered by airline pilots

Megan Fisher ${ }^{1 \star}$, Alexander Jolly ${ }^{2}$, Mumtaz Mooncey ${ }^{3}$,

Kerry Robinson ${ }^{1}$, Robert Lloyd ${ }^{1}$ and Dave Fielding ${ }^{2}$

${ }^{1}$ Whittington Health NHS Trust; ${ }^{2}$ Airline Captain and ${ }^{3}$ University

College London Hospital

${ }^{\star}$ Corresponding author.

doi: 10.1192/bjo.2021.388

Aims. To encourage multidisciplinary team learning by introducing Child \& Adolescent Mental Health (CAMHS) in-situ simulation training.

To provide focused Human Factors feedback through the expertise of senior airline pilots.

Method. The integration of the WingFactors in-situ simulation programme to multiple departments at Whittington Health NHS Trust has transformed the education landscape. The programme has received unanimously positive feedback, and the potential benefits for not only physical, but also mental health training, have been quickly recognised. A total of 90 simulations have been performed. A number of CAMHS scenarios have been designed with the primary aims of encouraging multidisciplinary training and increasing the focus on Human Factors in Psychiatry.

Simulation scenarios were performed in real clinical environments with primed actors, thus enabling high-fidelity in-situ simulation. Immediate 'hot' debriefs were delivered by clinical faculty and uniformed airline pilots, with emphasis on psychological safety to encourage participation from all team members. The key learning points were then detailed in written documents and circulated to the wider team as a valuable learning resource.

The first CAMHS simulation involved the acute management of a collapsed patient in the Emergency Department toilet, with a ligature tied around her neck and accompanied by a distressed patient. Another scenario addressed de-escalation techniques when dealing with a patient presenting with an overdose, who was threatening to leave the ward and posing potential risk to herself.

Result. The nature of these in-situ simulations enabled the multidisciplinary team to analyse practical considerations in the management of acute clinical situations. Scenarios were designed to focus on areas which had been identified as needing improvement for patient safety.

The observations provided by airline pilots increased the focus on Human Factors training. A number of key themes were identified, including the importance of effective team-briefing, distraction management and task allocation. This is of particular significance when managing a distressed patient and anxious relative, in a busy high-stress clinical environment.

Conclusion. In-situ simulation is a newly emerging concept in the field of Psychiatry, and the success of this programme has been highlighted through consistently positive feedback from participants, and nomination for the HSJ Award (Best Education Programme 2021). The involvement of airline pilots has promoted collaborative learning amongst the multidisciplinary team, and increased the focus on Human Factors in Psychiatry, clearly demonstrating the value of in-situ simulation training in this field.

\section{Novel approach to providing child and adolescent mental health education to allied health services}

\author{
Michael Foster ${ }^{1 \star}$ and Sally Arnold ${ }^{2}$ \\ ${ }^{1}$ The Darwin Centre and ${ }^{2}$ Harplands Hospital \\ ${ }^{*}$ Corresponding author.
}

doi: 10.1192/bjo.2021.389
Aims. To create and deliver a positive educational session for allied health services on prominent child and adolescent mental health conditions. It was hypothesised that delivering tailored teaching sessions on a range of child and adolescent mental health conditions would help improve the knowledge of allied health services. A quiz was administered at the beginning and end to assess the effectiveness of the sessions.

Background. In early 2019, a request was made by Staffordshire youth drug and alcohol service for an informal teaching session on prominent mental health conditions experienced by those under 18. The team often encountered the requested conditions but had no role in managing or treating them resulting in weaknesses in their knowledge. There was a strong desire to learn more about what the cause, presentation, diagnosis and management was of these too. An interactive, 60-75 minute session was requested on $\mathrm{ADHD}$, autism, depression, anxiety, emerging emotionally unstable personality disorder, bipolar affective disorder, and schizophrenia.

Method. Sessions were conducted at the local drug and alcohol service, and at 2 regional social services, in autumn 2019. A 21 question quiz, 3 questions on each topic, was taken at the start and end of each session. The quiz content was covered within the teaching session, as well as time for questions, then marked and converted into a percentage.

Result. 19 quizzes were taken; either by individuals or within pairs. The average score before the teaching was $43 \%$, increasing to an average of $90 \%$ after the teaching. The quiz showed good knowledge on anxiety and depression before the teaching, with an average pre-test score of $66 \%$, whereas knowledge on the other topics was less. Post-test scores increased to $100 \%$ for most areas, but scores for ASD and bipolar were both $66 \%$.

Conclusion. Feedback from the sessions was positive and staff across both services demonstrated a significant improvement in their understanding of prominent CAMHS mental health conditions. Further education and a change of approach to teaching is required for autism and bipolar affective disorder, both of which are challenging and broad topics.

The pre-teaching results do however demonstrate there is a need for better inter-agency education within teams, as well as reciprocal teaching so that knowledge from different teams can be shared. Further sessions are being proposed for other social services and general practises.

\section{Recruiting medical students from underrepresented backgrounds to a project to identify support challenges amongst their peers whilst encouraging early career engagement in psychiatry \\ Heather Gail McAdam ${ }^{1 \star}$ and Debbie Aitken ${ }^{2}$ \\ ${ }^{1}$ University of Edinburgh and ${ }^{2}$ University of Edinburgh, University of Cambridge \\ ${ }^{\star}$ Corresponding author.}

doi: 10.1192/bjo.2021.390

Aims. To engage lived experience individuals to run a project identifying the mental health challenges unique to medical students who self identify as belonging to marginalised groups;

To use the project findings to inform mental health support and education during medical training and beyond;

To encourage the individuals to engaged mental health policy and education whilst also using the process to inform their future medical careers, including in the field of psychiatry.

Method. Lived experience individuals were recruited to the project following open applications from medical students. The role 
was to design a project to create an evidence base for tailored support methods needed to reduce marginalised medical students' increased risk of poor mental health. This formed the basis of guidance on how medical schools and healthcare systems can improve wellbeing support for their students and staff. With support from a trained staff and student member, the recruited officers were encouraged to follow their preferred method for fulfilling the project aims, using their own and peers' experiences to inform what was most useful.

Result. Representatives were selected from BAME, LGBT+, international, disabled and widening participation backgrounds. The students decided to conduct a survey open to all medical student colleagues across the United Kingdom. The survey questions were split into four sections based on the challenges faced by their own lived experience: General Information; University and Community Experiences; Medical School Experiences and Teaching and Clinical Experiences. There were 58 questions in total including 26 multiple choice; 24 open answers; and 8 Likert scale.

Following data collection, the information taken from the survey and focus groups, supported by background reading, was thematically analysed to identify the key challenges. This will then be used to create a report to share with the medical school containing areas for improvement in mental health support, education and engagement. The officers themselves would also reflect on their experiences throughout the process, including their ability to engage in mental health policy, education and further career options such as psychiatry.

Conclusion. From creating an appropriate and supportive structure, it can be possible to encourage students with lived experience to share their challenges whilst becoming engaged in mental health policy and support. Furthermore, from creating a culture of reflection in the area of mental health, they are helping raise awareness of the subject early on in medical careers and promote engagement into specialties such as psychiatry.

Evaluating virtual role play based learning to improve the confidence and competence of Junior Doctors undertaking on call shifts in inpatient Psychiatry

\section{Katherine Gardner*}

Surrey and Borders Partnership NHS Foundation Trust ${ }^{\star}$ Corresponding author.

\section{doi: 10.1192/bjo.2021.391}

Aims. To enable junior doctors to practice their clinical skills in managing psychiatric emergencies via virtual role plays, and to gain confidence and competence in their skills in acute psychiatry. Lecture based learning about psychiatric emergencies is a part of the induction programme for all junior doctors starting their placements however practical learning and practice of skills in this area is not. The COVID-19 pandemic has further exacerbated this issue by providing an additional challenge to the delivery of face to face teaching for junior doctors both in clinical and educational settings.

Method. The author offered a virtual role play based teaching session to two cohorts of Junior Doctors (GP trainees and foundation trainees) who were starting their psychiatric hospital placements at Surrey and Borders Partnership. The virtual sessions were conducted over Microsoft teams. This session had been run once before as face to face teaching (F2F) in January $2019(\mathrm{~N}=9)$ prior to the COVID-19 pandemic. Data from this session were compared to data obtained from the virtual sessions in November 2020 and January $2021(\mathrm{~N}=16)$.
Pre and post study questionnaires were administered via Microsoft Forms. Each session lasted 1 hour and consisted of 3 different role play scenarios based around acute psychiatric emergencies. One junior doctor volunteer acted as the 'patient' in each scenario and another volunteer as the 'doctor'. The other participants all acted as observers. Each scenario lasted 10 minutes with ten minutes for feedback from the researcher afterwards using the ALOBA framework.

Categorical, ordinal data were collected using a Likert scale and general qualitative feedback was also gathered.

Result. The questionnaire return rate was $100 \%$ for F2F teaching and $57 \%$ for virtual teaching. $100 \%$ of participants felt that F2F role play was an acceptable way to practice skills in acute psychiatry vs $75 \%$ of participants who felt this about virtual role play. $100 \%$ of participants found that F2F role play was 'quite' or 'very' effective in improving their confidence and perceived competence in acute psychiatry vs $88 \%$ of participants who felt this about virtual role play.

Conclusion. Virtual role play based learning is an acceptable and effective method in improving the confidence and perceived competence of junior doctors undertaking on call shifts in inpatient psychiatry but it appears to be less effective than face to face role play based learning. The researcher will act upon the qualitative feedback obtained which suggested ways in which the virtual session could be improved.

\section{Foundation doctor preparedness for treating mental health conditions: results from a national survey}

George Gillett ${ }^{1 \star}$, Owen Davis ${ }^{2}$, Amarit Gill ${ }^{3}$ and Clare van Hamel $^{4}$

${ }^{1}$ IoPNN King's College London; ${ }^{2}$ UK Foundation Programme Leadership Fellow \& Foundation Year 2 Doctor, Oxford Foundation School; ${ }^{3}$ UK Foundation Programme Leadership Fellow \& Foundation Year 2 Doctor, Wales Foundation School and ${ }^{4}$ Severn Foundation School Director \& Clinical Advisor to UK Foundation Programme

${ }^{\star}$ Corresponding author.

doi: 10.1192/bjo.2021.392

Aims. Previous research suggests the prevalence of mental health conditions among medical inpatients may be as high as $38 \%$. Anecdotally, junior doctors report lacking the confidence, knowledge and skills to assess and treat patients with psychiatric conditions. Identifying this unmet need offers potential to improve standards of care and achieve parity of esteem between psychiatric and medical conditions within the general hospital. Aims:

To assess self-reported preparedness of newly-qualified Foundation Doctors to care for patients with acute or chronic psychiatric symptoms in comparison to physical health conditions. Method. In September of each year (2017, 2018, 2019), a survey was cascaded to all incoming Foundation Year 1 Doctors. For each respective year there were 1673, 961 \& 1301 respondents. Respondents were asked to rate their agreement with statements on a Likert scale. Statements pertaining to mental health included “a) I am competent in acute mental health care provision, b) I am competent in chronic mental health care provision" and "I feel confident in prescribing the following drugs; c) drugs for mental health problems". Comparison statements assessed confidence caring for medically unwell patients, performing practical procedures and prescribing drugs for physical health conditions.

Result. Preparedness for acute and chronic mental health were lower than both physical health comparison items; preparedness to care for patients with critical illness (acute: $\mathrm{r}=0.794, \mathrm{p}<$ 